Trapp, G.S.A., Giles-Corti, B., Christian, H.E., Timperio, A.F., McCormack, G.R., Bulsara, M.K. \& Villanueva, K.P. (2013). Driving down daily step counts: The impact of being driven to school on physical activity and sedentary behavior. Pediatric Exercise Science, 25(3), 337-346.

(C) 2013 Human Kinetics, Inc. as accepted for publication

This is pre-copy-editing, author-produced version of an article accepted for publication in Pediatric Exercise Science following peer review. The definitive published version (see citation above) is located on the article abstract page of the publisher, Human Kinetics.

This version was made available in the UWA Research Repository on 24 July 2014 in compliance with the publisher's policies on archiving in institutional repositories.

Use of the article is subject to copyright law. 


\title{
Driving down daily step counts: the impact of being driven to school on physical activity and
} sedentary behavior

\section{Running title:}

Car travel to school

\begin{abstract}
This study investigated whether being driven to school was associated with lower weekday and weekend step counts, less active out-of-school leisure pursuits and more sedentary behavior. Boys aged 10-13 years $(\mathrm{n}=384)$ and girls aged 9-13 years $(\mathrm{n}=500)$ attending 25 Australian primary schools wore a pedometer and completed a travel diary for one week. Parents and children completed surveys capturing leisure activity, screen-time and socio-demographics. Commute distance was objectively measured. Car travel was the most frequent mode of school transportation (boys: 51\%, girls 58\%). After adjustment (socio-demographics, commute distance, and school clustering) children who were driven recorded fewer weekday steps than those who walked (girls: -1393 steps $\mathrm{p}<0.001$, boys: -1569 steps, $\mathrm{p}=0.009$ ) and participated in fewer active leisure activities (girls only $\mathrm{p}=0.043$ ). There were no differences in weekend steps or screen time. Being driven to and from school is associated with fewer weekday pedometer-determined physical activity in 9-13 year-old elementary school children. Encouraging children, especially girls, to walk to and from school (even for part of the way for those living further distances) could protect the health and wellbeing of those children who are insufficiently active.
\end{abstract}

\section{Keywords}

Active school transport, walking, children 


\section{Introduction}

Despite the short journey between home and school, private motor vehicles are a predominant mode of school travel for many U.S. and Australian elementary school-aged children $(15,29)$. Discouraging car travel and promoting walking has been posited as a practical and convenient solution for increasing physical activity (28). Research to-date suggests that children who use passive school transportation modes (i.e. are driven) do less physical activity than children who use active modes (e.g., walking or cycling) $(2,8,12,17)$. However, failure to consider distance between home and school, travel mode misclassification, reliance on self-reported physical activity behavior, and inconsistent findings regarding the moderating effects of gender has limited the understanding of this relationship $(8,12)$. Furthermore, it is not clear whether inactive children are driven to school or whether the drive to school decreases children's physical activity per se. If sedentary behavior, out-of school active leisure pursuits and weekend physical activity levels do not differ between school commute modes then this may support the latter. Only two studies appear to have examined the relationship between school travel mode and sedentary behavior $(10,30)$ and found no association. However neither investigated whether gender moderated the relationship, despite established gender differences in sedentary behavior $(11,14,22)$. To better understand the contribution of school transportation to physical activity, this exploratory study examined the extent to which travel mode to school (i.e., being driven versus walking) was associated with weekday and weekend pedometer-determined physical activity, participation in active leisure outside school and sedentary behavior (i.e., screen-time) among boys and girls.

\section{Methods}

Cross-sectional data from 1291 Western Australian children taking part in the TRavel, Environment, and Kids (TREK) project were examined, the methods for which are presented fully elsewhere (9). Briefly, schools from low and high walkable areas and low, medium and high socio-economic strata were invited to participate and 25 agreed (69.4\%). Children from grades 5-7 were randomly selected to participate $(\mathrm{n}=1480$, 
56.6\%). Of those, 1291 provided pedometer data. Parents and children provided written informed consent.

The University of Western Australia's Human Ethics Committee provided ethics approval (RA/4/1/1394).

\section{School transportation mode}

Children completed a modified travel diary (10) during the monitoring period. Mode of transport to and from school (i.e., car, bus, walk, cycle, other) was recorded each day and the most frequent mode of travel computed (i.e., the mode used for $>50 \%$ of school trips). Participants with an even proportion (i.e., $50 \%$ each) of active and passive trips $(<5 \%)$ were coded according to the active travel mode.

\section{Socio-demographic variables}

Children reported their age, gender and school year and parents reported their highest level of education (i.e., completed high school or less, diploma/college/technical school, or university).

\section{Pedometer-assessed physical activity}

Accusplit AH120 pedometers (Accusplit, Inc., Livermore, CA, USA) captured children's step counts (27). The Accusplit AH120's multiday memory function eliminated the need for children to log step counts daily, improving data accuracy and completeness. The Accusplit AH120 is comparable in accuracy to the widely used Yamax SW series (Yamax Corp., Tokyo, Japan) (27) and has acceptable accuracy at walk speeds children walk to and from school $(>90 \mathrm{~m} / \mathrm{min})(13,27)$. Children wore the pedometer level with their hip bone in line with the midpoint of the right knee for seven consecutive days, except during water activities and while sleeping (distribution and collection days were excluded from analyses). Each morning children recorded their school travel mode and whether they had worn the pedometer the previous day. 


\section{Sedentary behavior}

Total screen-time was the sum of minutes/day the child spent using a computer or internet for pleasure, watching television/videos, and playing passive or active electronic games (parent-reported).

\section{Active leisure}

An active leisure index was estimated representing the count (0-7) of child-reported activities participated in outside of school in the last week (i.e., played in a park, playground, or playing field; played team sport; attended a club or youth group; gone for a walk in the neighborhood; played in the street; played in the yard; and took the dog for a walk).

\section{Commute distance to school}

The shortest distance (in meters) along the pedestrian network was calculated from each child's home address (parent-reported) to the 'access point' of the school boundary using Geographical Information Systems. Distance was collapsed into three categories $(\leq 800 \mathrm{~m} ; 801-1600 \mathrm{~m}$, and; $>1600 \mathrm{~m})$ as evidence suggests that one-way distances of $800 \mathrm{~m}$ and $1600 \mathrm{~m}$ are considered 'walkable' for children $(19,23,24)$.

\section{Statistical analysis}

Trips to school by bus or 'other' (i.e., scooter, rollerblades, skateboard) (both <1\%) were treated as car trips and cycle trips, respectively. Children who had $<3$ weekdays of valid pedometer data $(n=299,23 \%)$ or who cycled $>50 \%$ of school trips $(\mathrm{n}=108,8 \%)$ were excluded from analyses (pedometers perform poorly at 
quantifying activities that involve minimal vertical displacement) (1). A final sample of 884 cases were analyzed ( 50 cases=no distance to school data; 185 cases=no weekend pedometer steps). All results were stratified by gender and analyzed in 2011 using STATA IC 11. Chi-square analyses and independent t-tests were used to examine univariate associations for categorical variables (e.g., child sex) and continuous variables (e.g., weekday and weekend steps), respectively. Multivariate linear regression models estimated the association between driving versus walking to and from school and mean weekday and weekend pedometer steps/day, active leisure index, and minutes of screen time/day. Children who walked to and from school for $>50 \%$ of trips were the reference group.

\section{Results}

On average, children were $11.0( \pm 0.8)$ years old, took $12513( \pm 3489)$ steps/day on weekdays (boys $13523 \pm$ 3815; girls $11737 \pm 2997 ; p<0.001)$ and 8820 ( \pm 4407 ) steps/day on weekends (boys $9431 \pm 4934$; girls 8389 $\pm 3943, p=0.003$ ), spent $205( \pm 141)$ minutes/day in screen-based activity (boys $222 \pm 143$ minutes/day; girls $191 \pm 139$ minutes/day, $p=0.002)$ and participated in $3.6( \pm 1.6)$ active leisure activities/week outside school hours (boys $3.7 \pm 1.6$; girls $3.5 \pm 1.6, p=0.186$ ).

Children averaged $5.22( \pm 3.52)$ school trips/week by car $(3.07 \pm 3.40$ by foot and $0.33 \pm 1.00$ by bike).

Overall, $54.4 \%$ of boys and $57.4 \%$ of girls were driven for $>50 \%$ of trips to and from school. Children who were driven were more likely to live further away (boys and girls $p<0.05$ ), take fewer steps/day on weekdays (boys and girls $p<0.05$ ) and participate in fewer out-of-school active leisure activities (overall and girls $p<0.05$, boys $p>0.05$ ) compared with children who walked (Table 1). Being 'driven' to and from school was not associated with steps/day taken on weekends or minutes/day of screen time (Table 1). 
INSERT Table 1 here

After adjusting for demographic factors and commute distance to school (Table 2), boys who were driven took on average 1569 fewer steps/day on weekdays (95\% CI -2709, -429) than boys who walked representing an $11 \%$ difference in mean weekday steps, and girls who were driven took on average 1393 fewer steps/day on weekdays (95\% CI -2030, -756) compared with girls who walked representing a $12 \%$ difference in mean weekday steps. Girls, but not boys, who were driven participated in fewer out-of-school active leisure activities compared with those who walked (beta $-0.20,95 \% \mathrm{CI}-0.39,-0.01)$. There were no differences in steps/day accrued on weekends or daily duration of screen-time according to travel mode among either boys or girls (Table 2).

INSERT Table 2 here

\section{Discussion}

Consistent with previous studies $(3,7,16)$, children who were frequently driven to and from school undertook significantly fewer weekday steps than children who walked, even after adjusting for demographic factors and distance to school. However, previous studies did not adjust for distance to school - a consistent correlate of travel mode choice - and only one examined gender differences, whereby boys and girls step counts were similar (16). Studies using accelerometry have also found negative associations between passive school travel and physical activity levels $(4,20)$, although inconsistent findings in boys $(5$, 18) and girls (6). Our findings, along with others, suggest that both boys and girls will similarly benefit, in 
terms of higher levels of physical activity, from interventions that increase school-based active transportation.

No association was found between being driven to/from school and steps/day on weekends or minutes/day of screen time for boys or girls. This is consistent with previous studies that have also shown no association with weekend physical activity $(18,20-2)$ and sedentary behavior $(11,14,22)$ although this appears to be the first study to stratify results by gender. Whilst longitudinal research is needed, these results suggest that observed differences in weekday steps/day are not due to less active or inactive children being driven, but rather that being driven to and from school contributes to lower levels of physical activity in boys and girls. Thus decreasing the proportion of children who are driven to school is likely to result in modest net gains in children's physical activity.

Changing school travel patterns is a long-term commitment because of the embedded culture of car dependency. Importantly, strategies to promote active school travel involves removing barriers and increasing support, not least of which includes addressing family time constraints and allaying parental and child safety concerns by creating supportive environments around schools (i.e. walking/bicycle paths, reducing traffic volume and speed) and urban planning strategies (i.e. school siting decisions that reduce traffic exposure and improve street connectivity) $(9,25,26)$. For children whose only option is to be driven to school, replacing screen-time with physical activity is recommended.

\section{Limitations}

This study is limited by its cross-sectional design, the potential selection bias from $32 \%$ of the sample being excluded and pedometers not accurately measuring physical activity associated with cycling, walking at slow speeds (12), swimming and upper body movement. Also, dichotomising participants as being 'driven' or 'walked' may have resulted in a bias because there may have been differences in the behaviors of those 
who had an active mode $50 \%$ or more of the time compared with those who had an active mode each time.

Nevertheless, this study overcomes many common limitations of previous studies $(8,12,21)$ by using a travel diary during the physical activity monitoring period to classify children's school travel mode, including an objective measure of distance, examining differences in sedentary behavior, out-of-school leisure activities and weekend physical activity as well as weekday physical activity, and stratifying analyses by gender. Future research should incorporate longitudinal designs.

\title{
Conclusions
}

In conclusion, being driven to and from school is associated with reduced weekday pedometer-determined physical activity in 9-13 year-old elementary school children. Encouraging children, especially girls, to walk to and from school may help protect the health and wellbeing of those who are insufficiently active.

Consistent evidence across different cultures and school systems supports the need for public health and transportation policy initiatives to promote active school transport as a means of increasing children's weekday physical activity.

\author{
Abbreviations \\ TREK: TRavel, Environment and Kids Project
}

\section{References}

1. Bassett, D., Validity and reliability issues in objective monitoring of physical activity. Res.Q. Exerc. Sport. 71: 30-36, 2000. 
2. Chillón, P., F.B. Ortega, J.R. Ruiz, et al., Active commuting to school in children and adolescents: An opportunity to increase physical activity and fitness. Scand. J. Public. Health. 38(8): 873-879, 2010.

3. Constantinos, L.A. and R. Jago, Differences in physical activity by gender, weight status and travel mode to school in Cypriot children. Prev. Med. 47: 107-111, 2008.

4. Cooper, A.R., L.B. Andersen, N. Wedderkopp, A.S. Page, and K. Froberg, Physical activity levels of children who walk, cycle, or are driven to school. Am. J. Prev. Med. 29(3): 179-84, 2005.

5. Cooper, A.R., A.S. Page, L.J. Foster, and D. Qahwaji, Commuting to school: are children who walk more physically active? Am. J. Prev. Med. 25(4): 273-276, 2003.

6. Cooper, A.R., N. Wedderkopp, H. Wang, L.B. Andersen, K. Froberg, and A.S. Page, Active travel to school and cardiovascular fitness in Danish children and adolescents. Med. Sci. Sports. Exerc. 38(10): 1724-31, 2006.

7. Duncan, E.K., J.S. Duncan, and G. Schofield, Pedometer-determined physical activity and active transport in girls. IJBNPA. 5(2), 2008.

8. Faulkner, G.E.J., R.N. Buliung, P.K. Flora, and C. Fusco, Active school transport, physical activity levels and body weight of children and youth: A systematic review. Prev. Med. 48: 3-8, 2009.

9. Giles-Corti, B., G. Wood, T. Pikora, et al., School site and the potential to walk to school: The impact of street connectivity and traffic exposure in school neighbourhoods. Health Place. 17: 545$550,2011$.

10. Heelan, K.A., J.E. Donnelly, D.J. Jacobsen, M.S. Mayo, R. Washburn, and L. Greene, Active commuting to and from school and BMI in elementary school children - preliminary data. Child. Care. Health Dev. 341-349, 2005.

11. Kristjansdottir, G. and R. Vilhjalmsson, Sociodemographic differences in patterns of sedentary and physically active behavior in older children and adolescents. Acta Paediatrica. 90(4): 429-435, 2001. 
12. Lee, M., M. Orenstein, and M. Richardson, Systematic review of active commuting to school and children's physical activity and weight. J. Phys. Act. Health. 5: 930-949, 2008.

13. Mackett, R., B. Brown, Y. Gong, K. Kitazawa, and J. Paskins, Setting children free: children's independent movement in the local environment, in Working Papers Series2007, Centre for Advanced Spatial Analysis, UCL: London.

14. Matthews, C.E., K.Y. Chen, P.S. Freedson, et al., Amount of time spent in sedentary behaviors in the United States, 2003-2004. Am. J. Epidemiol. 167(7): 875-881, 2008.

15. McDonald, N.C., A.L. Brown, L.M. Marchetti, and M.S. Pedroso, U.S. school travel, 2009. An assessment of trends. Am. J. Prev. Med. 41(2): 146 -151, 2011.

16. Michaud-Tomson, L., M. Davidson, and T.F. Cuddihy, Walk to school - does it make a difference in children's physical activity levels? Achper. Aust. Healthy Lifestyles J. 50(3-4): 16-24, 2003.

17. Owen, C.G., C.M. Nightingale, A.R. Rudnicka, et al., Travel to school and physical activity levels in 9-10 year-old UK children of different ethnic origin; Child Heart and Health Study in England (CHASE). PloS one. 7(2): e30932, 2012.

18. Rosenberg, D.E., J.F. Sallis, T.L. Conway, K.L. Cain, and T.L. McKenzie, Active transportation to school over 2 years in relation to weight status and physical activity. Obesity Res. 14: 1771-1776, 2006.

19. Schlossberg, M., J. Greene, P. Phillips, B. Johnson, and B. Parker, School trips: effects of urban form and distance on travel mode. J. Am. Plann. Assoc. 72: 337-46, 2006.

20. Sirard, J.R., W.F. Riner, K.L. McIver, and R.R. Pate, Physical activity and active commuting to elementary school. Med. Sci. Sports Exerc. 37(12): 2062-9, 2005.

21. Sirard, J.R. and M.E. Slater, Walking and bicycling to school: a review. Am. J. Lifestyle Med. 2: 372396, 2008. 
22. te Velde, S.J., I.D. Bourdeaudhuij, I. Thorsdottir, et al., Patterns in sedentary and exercise behaviors and associations with overweight in 9-14-year-old boys and girls - a cross-sectional study. BMC Pub. Health. 7(16), 2007.

23. Timperio, A., K. Ball, J. Salmon, et al., Personal, family, social, and environmental correlates of active commuting to school. Am. J. Prev. Med. 30(1): 45-51, 2006.

24. Timperio, A., D. Crawford, A. Telford, and J. Salmon, Perceptions about the local neighborhood and walking and cycling among children. Prev. Med. 38(1): 39-47, 2004.

25. Trapp, G.S., B. Giles-Corti, H.E. Christian, et al., Increasing children's physical activity: individual, social, and environmental factors associated with walking to and from school. Health Educ.Behav.. 2011.

26. Trapp, G.S., B. Giles-Corti, H.E. Christian, et al., On your bike! A cross-sectional study of the individual, social and environmental correlates of cycling to school. IJBNPA. 8(123), 2011.

27. Trapp, G.S.A., Giles-Corti B., M. Bulsara, et al., Measurement of children's walking using a pedometer with a built-in memory. J. Sci. Med. Sport. 2012.

28. Tudor-Locke, C., B.E. Ainsworth, and P.M. Barry, Active commuting to school: an overlooked source of children's physical activity? Sports Med. 31(5): 309-313, 2001.

29. van der Ploeg, H., D. Merom, G. Corpuz, and A. Bauman, Trends in Australian children travelling to school 1971-2003: burning petrol or carbohydrates? Prev. Med. 46(1): 60-2, 2008.

30. Wen, L.M., D. Merom, C. Rissel, and J.M. Simpson, Weight status, modes of travel to school and screen time: a cross-sectional survey of children aged 10-13 years in Sydney. Health Promot. J. Aust. 21(1): 57-63, 2010. 
Table 1: Bivariate associations of travelling to and from school by car or walking $(\mathbf{n}=\mathbf{8 8 4})$

\begin{tabular}{cccccc}
\hline \multicolumn{2}{c}{ All Children $(\mathrm{n}=884)$} & \multicolumn{2}{c}{ Boys $(\mathrm{n}=384)$} & \multicolumn{2}{c}{ Girls $(\mathrm{n}=500)$} \\
\hline Walked & Driven & Walked & Driven & Walked & Driven \\
$(\mathrm{n}=388)$ & $(\mathrm{n}=496)$ & $(\mathrm{n}=175)$ & $(\mathrm{n}=209)$ & $(\mathrm{n}=213)$ & $(\mathrm{n}=287)$
\end{tabular}

\section{Demographics (\%)}

Sex of child - female

54.9

57.9

School Year

Year 5

Year 6

Year 7

Highest maternal

education

Less than

Secondary

22.1

28.1

16.1

28.6

26.9

27.8

58.9

54.7

62.1

21.7

14.3

16.8

19.2

19.0

17.1

21.7

57.1

56.3

53.0

$\underline{\text { Distance to school }^{\mathrm{a}}}$

$\begin{array}{lcccccc}\text { Mean meters (SD) } & \mathbf{7 9 1} & \mathbf{2 5 8 2} & \mathbf{9 5 9} & \mathbf{2 7 6 4} & \mathbf{6 5 2} \\ & (\mathbf{1 3 7 5}) & (\mathbf{2 9 3 5}) * * * & \mathbf{( 1 8 6 1 )} & \mathbf{( 3 1 7 6 ) * * *} & & (\mathbf{2 7 4 9}) * * * \\ & \mathbf{7 2 . 4} & \mathbf{2 3 . 0} * * * & \mathbf{6 8 . 3} & \mathbf{1 9 . 6} * * * & \mathbf{7 5 . 7} & \mathbf{2 5 . 5} * * * \\ \text { 0 and 800m } & \mathbf{1 9 . 2} & \mathbf{2 4 . 1} & \mathbf{2 0 . 4} & \mathbf{2 3 . 7} & \mathbf{1 8 . 3} & \mathbf{2 4 . 4} \\ 801 \text { and } 1600 \mathrm{~m} & \mathbf{8 . 4} & \mathbf{5 2 . 9} & \mathbf{1 1 . 4} & \mathbf{5 6 . 7} & \mathbf{5 . 9} & \mathbf{5 0 . 2}\end{array}$

Physical activity 


\begin{tabular}{|c|c|c|c|c|c|c|}
\hline Mean weekday steps & 13310 & 11889 & 14525 & 12684 & 12312 & 11310 \\
\hline (SD) & (3498) & $(3355)^{* * *}$ & (3628) & $(3774) * * *$ & (3053) & $(2886) * *$ \\
\hline Mean weekend steps ${ }^{\mathrm{b}}$ & 9036 & & 9550 & & 8657 & \\
\hline (SD) & (4197) & $8656(4558)$ & (4620) & $9337(5183)$ & (3825) & 8192 (4024) \\
\hline
\end{tabular}

Active leisure index

(SD)
$3.8(1.7)$
$3.5(1.5)^{* * * *}$
$3.8(1.7)$
3.5 (1.4)
$3.7(1.7) \quad 3.4(1.5) *$

$\underline{\text { Sedentary behavior }}$

$\underline{(\%)}$

Mean screen time

(minutes/day) (SD)

207.0

216.8

227.5

198.7

185.3

(145.2)

(142.9)

(143.3)

(146.9)

$* p \leq 0.05 ; * * p \leq 0.01 ; * * * p \leq 0.001$

${ }^{a} \mathrm{n}=834$ cases ( 361 males and 473 females) who had provided a home address

${ }^{b} \mathrm{n}=699$ cases ( 289 boys and 410 girls) who had valid pedometer data for at least one weekend day 
Table 2: Linear regression results of being driven to and from $\operatorname{school}^{\mathbf{a}}(\mathbf{n}=\mathbf{8 8 4})$

\begin{tabular}{llllll}
\multicolumn{4}{c}{ Model 1 $^{\mathrm{b}}$} & \multicolumn{3}{c}{ Model 2 $^{\mathrm{c}}$} \\
$\beta$ & $95 \% \mathrm{CI}$ & $P$ & $\beta$ & $95 \% \mathrm{CI}$ & $P$
\end{tabular}

\section{Physical activity}

Mean weekday steps

$\begin{array}{lrrrrrr}\text { All children } & -1413 & -2051,-776 & 0.000 & -1471 & -2126,-817 & 0.000 \\ \text { Boys } & -1744 & -2717,-771 & 0.001 & -1569 & -2709,-429 & 0.009 \\ \text { Girls } & -1152 & -1815,-488 & 0.001 & -1393 & -2030,-756 & 0.000\end{array}$

Mean weekend steps

$\begin{array}{lcccccc}\text { All children } & -419 & -1063,225 & 0.192 & -470 & -1135,195 & 0.158 \\ \text { Boys } & 28 & -1094,1149 & 0.959 & -251 & -1509,1006 & 0.684 \\ \text { Girls } & -659 & -1356,37 & 0.062 & -599 & -1453,255 & 0.160\end{array}$

Active Leisure index

$\begin{array}{lcccccc}\text { All children } & \mathbf{- 0 . 2 5} & \mathbf{- 0 . 4 6 , - 0 . 0 4} & \mathbf{0 . 0 2 0} & \mathbf{- 0 . 2 4} & \mathbf{- 0 . 4 5 ,}, \mathbf{- 0 . 0 3} & \mathbf{0 . 0 2 5} \\ \text { Boys } & -0.26 & -0.63,0.11 & 0.154 & -0.33 & -0.75,0.10 & 0.128 \\ \text { Girls } & \mathbf{- 0 . 2 7} & \mathbf{- 0 . 5 1 , - 0 . 0 3} & \mathbf{0 . 0 3 0} & \mathbf{- 0 . 2 0} & \mathbf{- 0 . 3 9 ,}, \mathbf{- 0 . 0 1} & \mathbf{0 . 0 4 3}\end{array}$

\section{$\underline{\text { Sedentary behavior }}$}

Mean screen time

(minutes/day)

$\begin{array}{lcccccc}\text { All children } & -6.75 & -24.68,11.17 & 0.444 & -11.60 & -31.98,8.77 & 0.251 \\ \text { Boys } & -0.87 & -30.12,28.38 & 0.952 & 3.59 & -27.60,34.78 & 0.814 \\ \text { Girls } & -12.49 & -37.66,12.68 & 0.316 & -24.9 & -55.08,5.26 & 0.101\end{array}$

${ }^{a}$ Defined as being driven more than half of all school trips to and from school (reference category $=$ walking)

${ }^{\mathrm{b}}$ Adjusted for school clustering and demographic factors (i.e., sex, child's grade, maternal education)

${ }^{\mathrm{c}}$ Adjusted for school clustering, demographic factors and distance to school 
286 